\title{
IDH1/2 mutation status combined with Ki-67 labeling index defines distinct prognostic groups in glioma
}

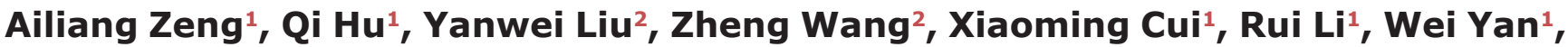 \\ Yongping You ${ }^{1}$ \\ ${ }^{1}$ Department of Neurosurgery, The First Affiliated Hospital of Nanjing Medical University, Nanjing 210029, PR China \\ ${ }^{2}$ Beijing Neurosurgical Institute, Capital Medical University, Beijing 100050, PR China \\ Correspondence to: \\ Yongping You, e-mail: yypl9@njmu.edu.cn \\ Wei Yan, e-mail: neuro_yw@njmu.edu.cn \\ Keywords: IDHI/2 mutation, Ki-67 labeling index, prognosis, glioma, molecular classification \\ Received: March 24, $2015 \quad$ Accepted: August 07, $2015 \quad$ Published: August 18, 2015
}

\section{ABSTRACT}

The current World Health Organization (WHO) classification of human gliomas is mainly based on morphology. However, it has limitations in prognostic prediction. We examined whether combining isocitrate dehydrogenase (IDH) 1 / 2 mutation status with the Ki-67 labeling index would improve the definition of prognostically distinct entities. We investigated the correlation of Ki-67 expression with IDH1/ 2 mutation status and their impact on clinical outcome in 703 gliomas. Low Ki-67 expression closely overlapped with IDH1/ 2 mutation in our cohort $(P<0.0001)$. Patients with IDH1/2 mutation survived significantly longer than patients with wild-type IDH1/2 did $(P<0.0001)$; higher Ki-67 expression was associated with shorter progressionfree survival and overall survival (OS) $(P<0.0001)$. IDH1/ 2 combined with Ki-67 was used to re-classify glioma patients into five groups. IDH1/2 mutant patients with low and moderate Ki-67 expression (Group1) had the best prognosis, whereas patients with wild-type IDH1/2 and high Ki-67 expression (Group5) had the worst prognosis (Median OS $=1527$ vs. 355 days, $P<0.0001$ ). To summarize, our new classification model distinguishes biologically distinct subgroups and provides prognostic information regardless of the conventional WHO grade. Classification based on IDH1/ 2 mutation status and Ki-67 expression level could be more convenient for clinical application and guide personalized treatment in malignant gliomas.

\section{INTRODUCTION}

Diffuse gliomas represent a biologically heterogeneous group of primary brain tumors [1]. Their intrinsically invasive characteristic encumbers complete surgical resection, mandating the development of more effective medical therapies [2]. World Health Organization (WHO) grade I gliomas are often curable with definitive surgical resection [3]; WHO grade II, III, and IV gliomas are malignant, diffuse, and with poor outcome; glioblastoma (WHO grade IV, the most invasive grade) is characterized by astrocytic morphology with induced angiogenesis and/ or necrosis. The ability of the current WHO classification for predicting human glioma diagnosis and prognosis remains limited $[4,5]$. In the past 30 years, many molecules have been implicated in the grading of prognostically different astrocytic tumors [6]. In 2009, it was determined that isocitrate dehydrogenase (IDH) 1/2 mutations were correlated with a prognostically improved adult glioma subtype. IDH1 mutation affects amino acid 132 of the IDH1 gene. The analogous amino acid (R172) of the IDH2 gene is often affected in tumors without IDH1 mutations. IDH1 mutation is involved in $>70 \%$ of astrocytomas, oligodendrogliomas, and secondary glioblastomas [3]. IDH mutant tumors have distinctive genetic and clinical features, and patients with such mutations have better outcome than those with wild-type IDH $[3,7]$. Ki-67 is a reliable indicator of cancer cell proliferation activity and is used for routine clinical investigation, and it predicts worse prognosis for patients with glioma [7-9]. Recent studies have defined the molecular classification of gliomas for clinical use [4, 7], but we still look forward to a more convenient method for predicting prognosis based on IDH1/2 mutation status and Ki-67 expression level. 
Herein, we explored IDH1/2 mutation (IDH1/2 mut) and its association with $\mathrm{Ki}-67$ expression in primary tumor samples from a large Chinese Glioma Genome Atlas (CGGA) cohort of 703 gliomas (see Supplementary Table S1). The independent prognostic impact of the two biomarkers on patients with glioma [9, 10] was validated using survival analysis. Then, we applied the two biomarkers to predict diagnosis and prognosis in our glioma classification model.

\section{RESULTS}

\section{Both IDH1/2 mutation and Ki-67 expression level were important prognostic factors of gliomas}

Recent investigations implicated IDH1/2 mutation in the classification of biologically distinct groups of gliomas, and indicated improved outcome [11-13]. Therefore, we used Kaplan-Meier curves to evaluate the outcome of 703 patients with glioma following stratification by IDH1/2 mutation status (Figure 1A). IDH mut was associated with longer survival relative to wild-type IDH (IDH1/2 wt) (Median overall survival, OS $=1410$ vs. 461 days; log-rank test, $P<0.0001)$. Previously [7, 8], we had classified Ki67 expression in patients into three levels (low, moderate, high). High Ki-67 expression was strongly associated with shorter progression-free survival (PFS) and OS as compared to moderate $\mathrm{Ki}-67$ expression (Median OS = 381 vs. 619 days). Moderate Ki-67 expression had worse prognosis than low Ki-67 expression (Median OS = 619 vs. 1592 days; log-rank test, $P<0.0001)$. Overall, higher Ki-67 expression
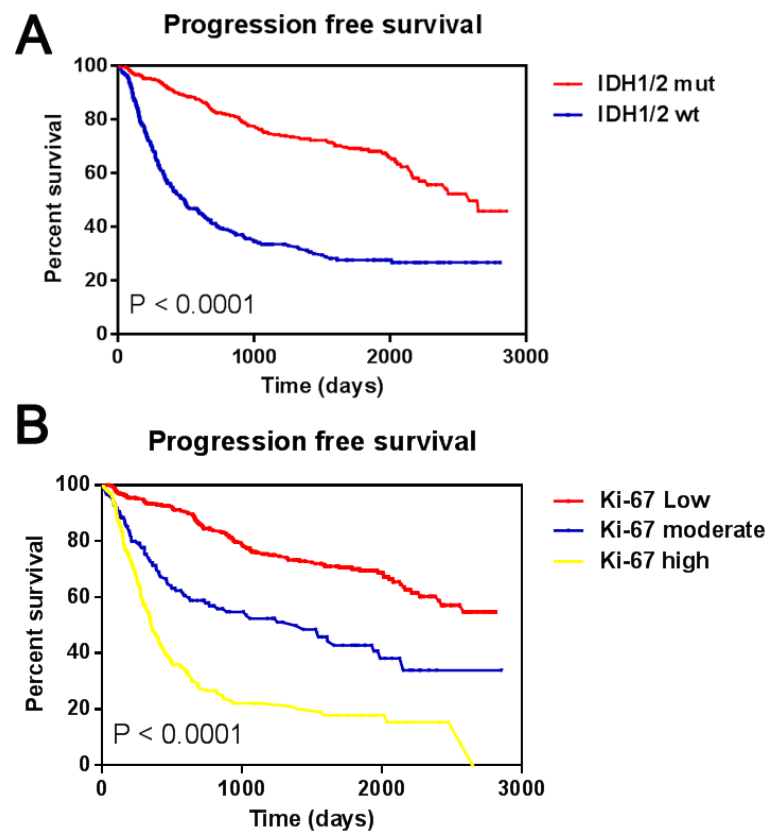

was associated with shorter PFS and OS (Figure 1B). Taken together, both IDH1/2 mutation and Ki-67 expression level are reliable prognostic markers of gliomas.

\section{Decreased Ki-67 expression was prevalent in IDH1/2 mut gliomas}

There were 381 samples in the IDH1/2 mut group, which included 250, 79, and 52 low- moderate-, and highKi-67 expression gliomas, respectively. The IDH1/2 wt group ( $n=322$ samples), was comprised of 89,78 , and 155 lowmoderate-, and high-Ki-67 expression gliomas, respectively. Figure 2 shows that $65.62 \%(250 / 381)$ and $27.64 \%$ (89/322) of the IDH1/2 mut and IDH1/2 wt groups, respectively, expressed low Ki-67 $(P<0.0001$, chi-square test) (Figure 2). The distribution of gliomas with high Ki-67 expression was the inverse of this. The proportion of high $\mathrm{Ki}-67$ expression in the IDH1/2 mut and IDH1/2 wt group was $13.65 \%$ $(52 / 381)$ and $48.14 \%(155 / 322)$, respectively $(P<0.0001$, chi-square test). No statistical significance was detected in the distribution of moderate Ki-67 between the IDH1/2 mut and IDH1/2 wt groups ( $P=0.378$, chi-square test). These findings confirm a strong correlation between IDH mutation status and $\mathrm{Ki}-67$ expression level, suggesting that low Ki-67 expression is characteristically present in gliomas with IDH mutation.

\section{Patients with low Ki-67 expression did not survive significantly longer than moderate Ki-67 expression in IDH1/2 mutant group}

Olar demonstrated a statistical interaction between IDH mutation and the mitotic index, suggesting that the

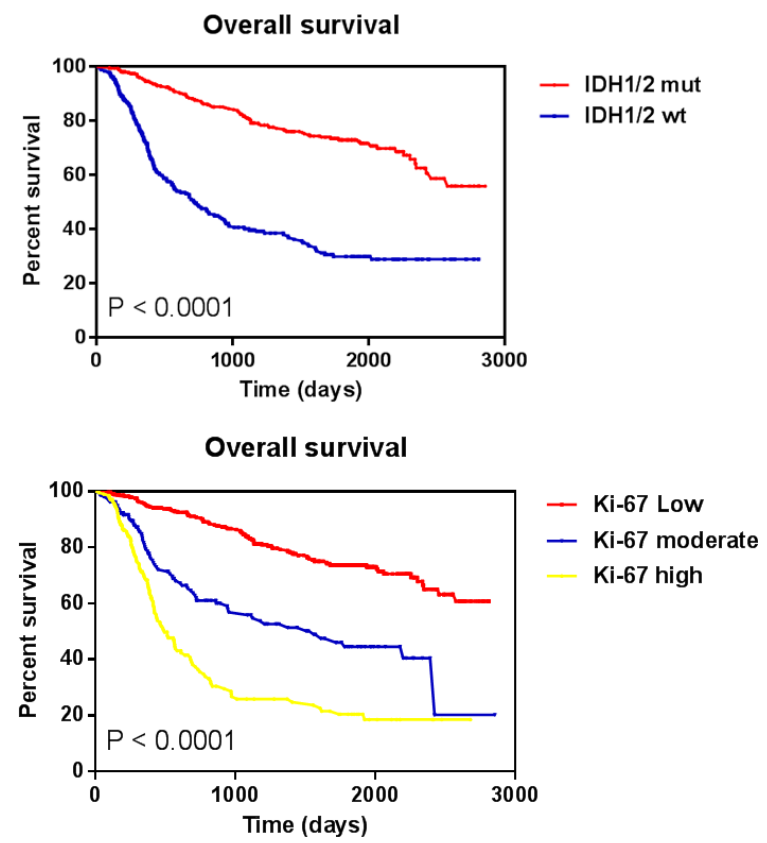

Figure 1: Progression free survival and overall survival among all 703 gliomas. A. Progression free survival and overall survival among all 703 gliomas stratified by IDH1/2 mutant status; B. Progression free survival and overall survival among all 703 gliomas stratified by relative Ki-67 expression level. 
A

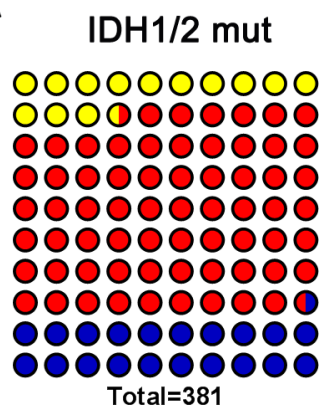

B

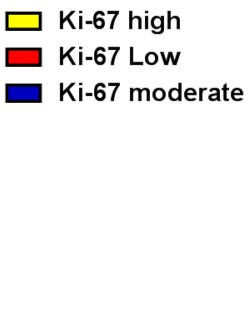

IDH1/2 wt

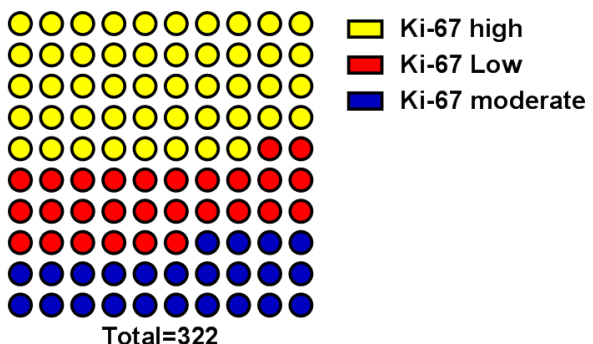

Figure 2: Distribution of low, moderate and high Ki-67 expression among IDH1/2 mut and IDH1/2 wt group. A. Ki-67 expression was determined in IDH1/2 mut group; B. Ki-67 expression was determined in IDH1/2 wt group.
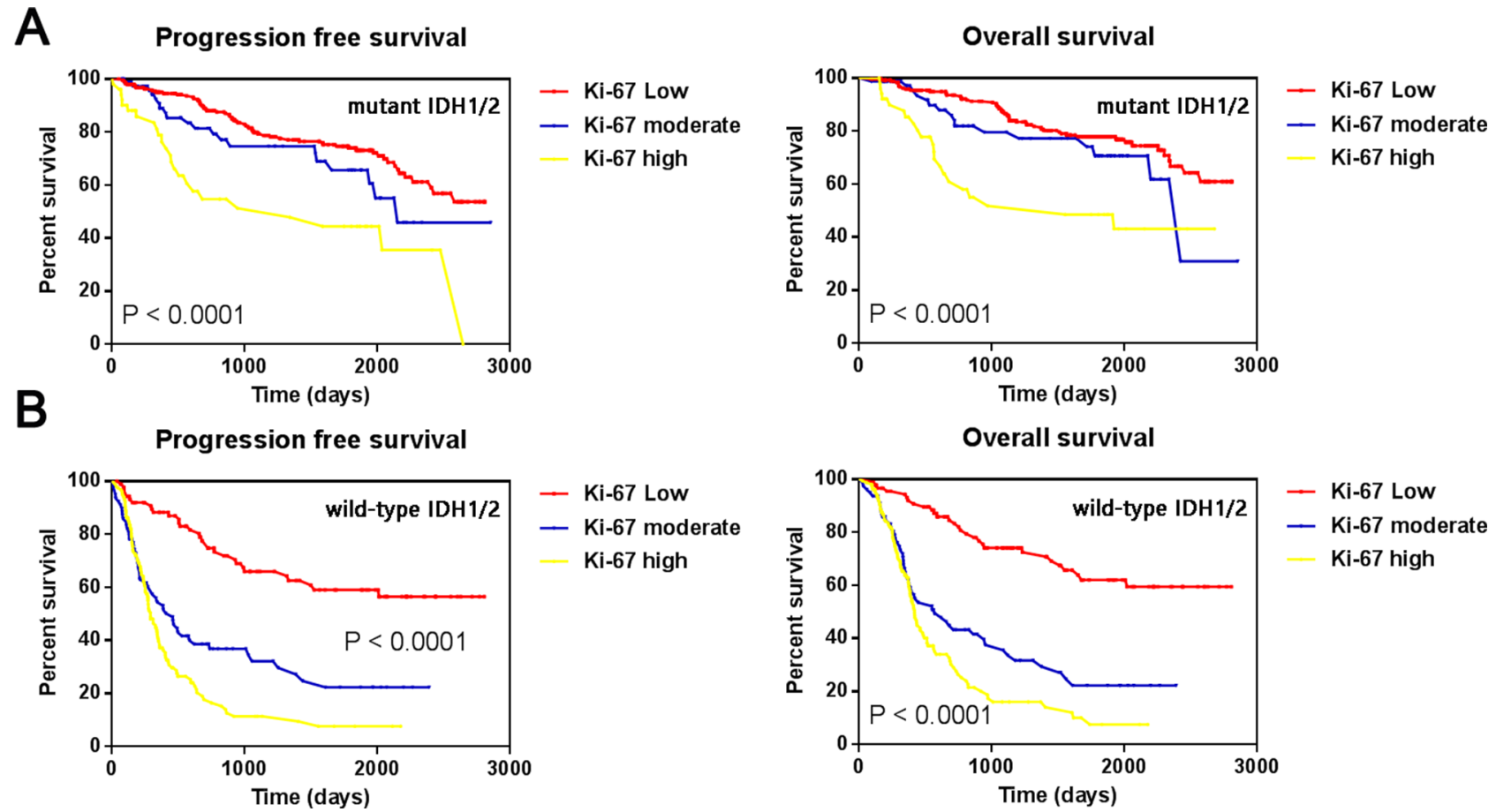

Figure 3: Kaplan-Meier estimates of survival for all 703 gliomas. A. Progression free survival and Overall survival among IDH1/2 mutant tumors stratified by relative Ki-67 expression level; B. Progression free survival and Overall survival among IDH1/2 wild type tumors stratified by relative Ki-67 expression level.

impact of cellular proliferation on clinical outcome was dependent on IDH mutation status in patients with gliomas [4]. To further understand the effect of Ki-67 expression on prognoses among different IDH mutation statuses, we classified the 703 samples as IDH1/2 mut (Figure $3 \mathrm{~A}$ ) or IDH1/2 wt (Figure 3B). As expected, higher Ki67 expression in the IDH wt tumors was associated with shorter PFS and OS (Median OS of high, moderate and low Ki-67 = 355, 434, and 1234 days, respectively; logrank test, $P<0.0001)$. Among the IDH mut tumors, high Ki-67 expression was strongly associated with the shortest PFS and OS (median OS = 566 days; log-rank test, $P<0.0001)$; interestingly, the prognosis of the low and moderate Ki-67 expression groups were not statistically different (median OS $=1699$ and 786 days, respectively; log-rank test, $P=0.158)$. Notably, low Ki67 expression did not necessarily predict better prognosis than moderate $\mathrm{Ki}-67$ expression among the IDH1/2 mut gliomas (Figure 3A).

\section{IDH1/2 mutation could be used to sub-classify gliomas in combination with Ki-67 expression}

To further detail the glioma molecular classification, we designed a glioma classification model based on IDH1/2 mutation status and Ki-67 expression level (Figure 4). We classified gliomas as IDH1/2 mut or IDH $1 / 2 \mathrm{wt}$, and then designated IDH1/2 mut with high Ki-67 expression as Group 2, IDH1/2 wt with low Ki67 expression as Group 3, IDH1/2 wt with moderate 
Ki-67 expression as Group 4, and IDH1/2 wt with high Ki-67 expression as Group 5. IDH1/2 mut with low and moderate Ki-67 expression were combined into Group 1 due to their similar prognoses, and accounted for $86.35 \%$ (329/381) of patients with IDH1/2 mutation and $46.80 \%$ $(329 / 703)$ of all recruited patients.

As shown in Figure 5, Group 1 patients had the longest PFS and OS, whereas Group 5 patients had the shortest (median OS $=1527$ vs. 355 days; log-rank test, $P<0.0001)$. These findings confirm the premise that different glioma oncobiology, such as different IDH1/2 mutation status and Ki-67 expression levels, are associated with discriminating prognosis [4]. However, patients with IDH1/2 wt gliomas and low Ki-67 expression survived longer than those with IDH1/2 mut gliomas and high Ki-67 expression (Median OS $=1234$ vs. 566 days), which contradicts the findings in Figure 1A. Patients with IDH1/2 mut gliomas and high Ki-67 expression survived longer than did those with IDH1/2 wt gliomas and moderate Ki-67 expression (Median OS $=566$ vs. 434 days), which contradicts the findings in Figure 1B. Therefore, we conclude that classification combining IDH mutation with Ki-67 expression levels could represent a more precise biological property and prognosis compared to classification using IDH mutation status or Ki-67 expression level separately.

\section{DISCUSSION}

IDH1/2 mutations were first reported in 2009, occurring in about $80 \%$ of grade II-III gliomas and secondary glioblastoma multiforme (GBM) [13]. IDH1/2 mutations are early and common events in the etiopathology of astrocytomas, oligodendrogliomas, and oligoastrocytomas, and they are associated with increased DNA methylation, and termed the glioma-CpG

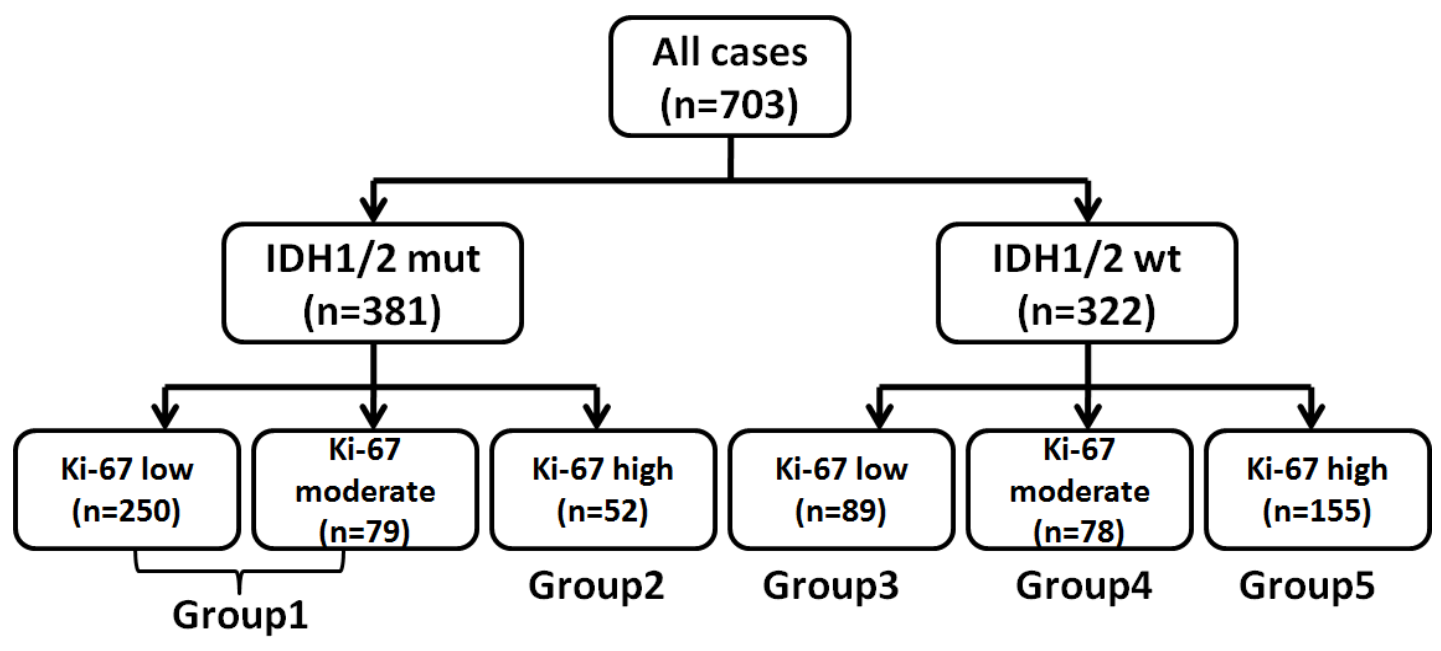

Figure 4: Model for classification of gliomas based on molecular markers. IDH1/2 mutant tumors with low and moderate Ki67 expression was termed as Group 1, IDH1/2 mutant tumors with high Ki-67 expression as Group 2, IDH1/2 wt tumors with low Ki-67 expression as Group 3, IDH wt tumors with moderate Ki-67 expression as Group 4, and IDH wt tumors with high Ki-67 expression was defined as Group 5.

A

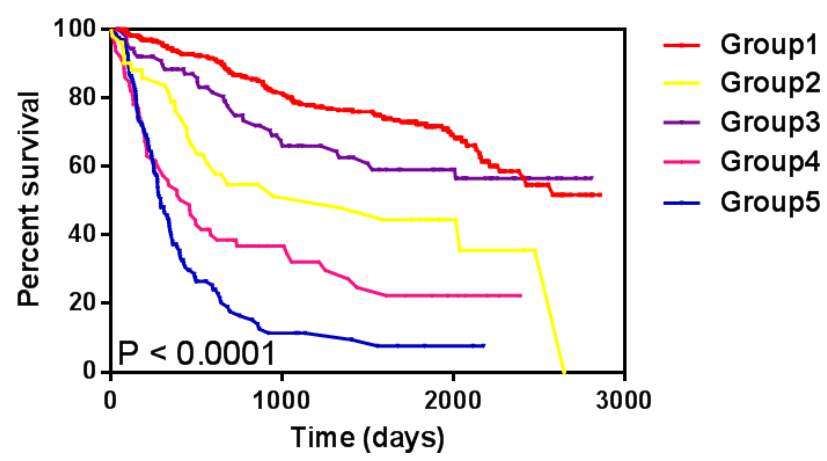

B Overall survival

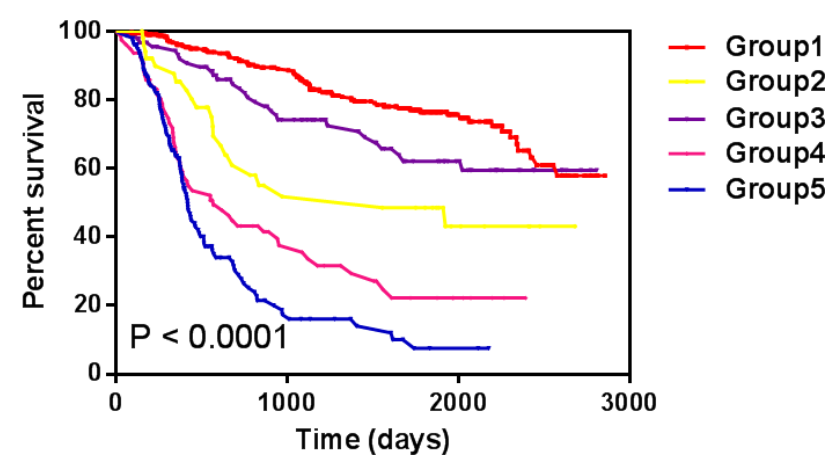

Figure 5: Progression free survival and overall survival among the new five groups. A. Progression free survival among the new five groups stratified by combined IDH mutation and Ki-67 expression status; B. Overall survival among the new five groups stratified by combined IDH mutation and Ki-67 expression status. 
island methylator phenotype (G-CIMP) [4]. Wild-type IDH produces $\alpha$-ketoglutarate, whereas mutant IDH1/2 encodes mutant IDH1/2 proteins. Mutant IDH1/2 proteins have altered substrate specificity and they produce more D-2-hydroxyglutarate, which acts as an oncometabolite [14-16]. Acquisition of IDH1 mutations, followed by compatible molecular changes such as TP53 mutation or $1 \mathrm{p} / 19 \mathrm{q}$ codeletion in a common tumor progenitor cell, can lead to gliomagenesis [17]. Although IDH1/2 mutations might not directly trigger tumorigenesis, they increase the risk of other tumor-promoting mutations that cooperate with IDH mutations to induce gliomagenesis [18]. IDH mutation status is stable, while additional molecular events, such as allelic loss of $1 p / 19 q(L O H ~ 1 p / 19 q)$ or TP53- mutations, accumulate during the progression of low-grade glioma to secondary high-grade glioma [19]. Clinically, IDH mut tumors are associated with longer OS as compared to IDH wt tumors among most glioma entities $[4,7]$. Hartmann et al. reported that glioblastoma patients with mutant IDH had better outcomes than that of patients with grade III anaplastic astrocytoma with wild-type IDH [11]. All this suggests that IDH mutations correlate with glioma etiopathology and act as a powerful prognostic factor among patients with gliomas. It is not surprising that current findings suggest that glioma subtypes can be separated following stratification by IDH mutation status $[11,14]$. In comparison with IDH1/2 mutations being widely considered a key development in the early stage of astrocytic tumors, increasing Ki-67 expression is considered the terminal event in glioma progression $[8,9,20,21]$. $\mathrm{Ki}-67$ is a nuclear protein and is frequently considered an indicator of cellular proliferation [22]. Similar to IDH mutation, Ki-67 expression is also an independent prognostic factor in glioma [9]. Recent work has reported that high $\mathrm{Ki}-67$ expression is dominant in IDH1/2 wt gliomas [7] and that low Ki-67 expression is associated with IDH1 mutations in primary GBMs [20]. These findings demonstrate the relationship between $\mathrm{Ki}$ 67 expression and IDH1/2 mutations in gliomas.

We classified 703 gliomas according to IDH mutation status and $\mathrm{Ki}-67$ expression level regardless of morphological grading. As shown in Supplementary Table S2, we examined groups based on IDH/Ki-67 and WHO grades in multivariate Cox proportional hazard regression analyses, and we found that IDH/Ki67 could define novel prognostic groups independent of WHO grade. Our molecular classification model detected significantly different prognoses among the five subtypes. Group 1 patients had the best prognosis, whereas Group 5 patients had the worst. That low Ki67 expression did not differ significantly from moderate Ki-67 expression in Group 1 in terms of clinical outcome could be attributed to the larger effect size of IDH mutation on prognosis among IDH mut tumors. Given the majority of samples with low $(n=250)$ and moderate $(n=79) \mathrm{Ki}-67$ expression among the IDH mut tumors, this effect appeared relatively robust. We also found that patients with IDH1/2 mut gliomas and high Ki-67 expression (Group 2) had worse clinical outcome than did patients with DH1/2 wt gliomas and low Ki-67 expression (Group 3). We speculate that the flourishing cellular proliferation activity in Group 2 contributed to the worse prognosis.

Cai et al. used ATRX mRNA expression combined with IDH1/2 mutation status and $\mathrm{Ki}-67$ expression to refine the molecular classification of 169 astrocytic tumors [7]. Olar et al. employed IDH1/2 mutation combined with $1 \mathrm{p} / 19 \mathrm{q}$ codeletion and the mitotic index to categorize 558 grade II-III diffuse gliomas [4]. We combined IDH1/2 mutation status with Ki-67 expression level to describe the biological properties and prognosis for each patient quickly and precisely in 703 gliomas.

In summary, our results demonstrate that combining IDH1/2 mutation status with Ki-67 expression levels can be used to define five glioma subgroups regardless of the conventional WHO grade. This approach further characterizes the distinct glioma biological properties and clinical outcome and can act as a complementary description to conventional glioma classification.

\section{MATERIALS AND METHODS}

\section{Patients and samples}

All glioma samples included in our study were from the Chinese Glioma Genome Atlas (CGGA). The patients underwent surgical resection between January 2006 and December 2010. Patients were eligible for the study if the diagnosis of glioma was established histologically according to the 2007 WHO classification. Tumor tissue samples were obtained by surgical resection before treatment with radiation and chemotherapy. This study was approved by the institutional review boards of the hospitals, and written informed consent was obtained from all patients. The following data were collected: WHO grade (as per original pathology report confirmed with H\&E central review per current 2007 WHO grading criteria), survival status (alive or dead), IDH1/2 mutational status, Ki-67 expression, overall survival (OS), and progression-free survival (PFS). Data sets are provided in Supplementary Table S1.

\section{DNA pyro-sequencing for IDH1/2 mutation}

Pyro-sequencing was performed as ref [7]. Briefly, genomic DNA was extracted from frozen tissues with a QIAamp DNA Mini Kit (Qiagen) according to the manufacturer's protocol. DNA concentration and quality were measured using a Nano-Drop ND-1000 spectrophotometer (NanoDrop Technologies, Houston, TX). Pyrosequencing of IDH1/2 mutations was supported by Gene-tech (Shanghai, China) and performed on a Pyro-Mark Q96 ID System (Qiagen, Valencia, Calif). 
The primers 5'-GCT TGT GAG TGG ATG GGT AAA AC-3', 5'-Biotin-TTG CCA ACA TGA CTT ACT TGA TC- $3^{\prime}$ for IDH1 and $5^{\prime}$-ATC CTG GGG GGG ACT GTC TT-3', 5'- Biotin-CTC TCC ACC CTG GCC TAC CT$3^{\prime}$ for IDH2 were used for PCR amplification, and the primers 5'-TGG ATG GGT AAA ACC T-3' for IDH1 and 5'-AGC CCA TCA CCA TTG-3' for IDH2 were used for sequencing.

\section{Immunohistochemistry}

Immunohistochemistry was performed according to our previous report $[7,8]$. Anti-ki-67 at a dilution of 1:100. The staining intensity was jointly scored by two pathologists without knowledge of clinical information on a scale of $0-3$, with $0=$ no or rare occurrence of staining, $1 \leq 10 \%$ of cells positively stained, $2=10-30 \%$ of cells positively stained, $3 \geq 30 \%$ of cells positively stained. Score $0-1$, Score 2 and Score 3 indicated low, moderate and high expression of Ki-67. Controls without primary antibody and positive control tissues were included in all experiments to ensure the quality of staining.

\section{Statistical analysis}

Two clinical end-points were used to measure clinical outcome, progression-free survival (PFS) and overall survival (OS). PFS was defined as the time interval between the date of surgery and the date of first recurrence. OS was defined as the time interval between the date of surgery and the date of death. Kaplan-Meier survival analysis was used to estimate the survival distributions, and the log-rank test was used to assess the statistical significance between stratified survival groups using GraphPad Prism 5.0 statistical software. A two-sided $P$ value of $<0.05$ was regarded as significant.

\section{ACKNOWLEDGMENTS}

This work was supported by the following grants: 1. National Natural Science Foundation of China (No. 81472362 and 81402056); 2. National High Technology Research and Development Program of China (863) (No. 2012AA02A508); 3. International Cooperation Program (No. 2012DFA30470).

\section{FUNDING}

This work was supported by grants from the National Natural Science Foundation of China (No. 81402056 and 81472362), National High Technology Research and Development Program of China (863) (No. 2012AA02A508), International Cooperation Program (No. 2012DFA30470).

\section{CONFLICTS OF INTEREST}

None of the authors has any conflict of interest to disclose.

\section{REFERENCES}

1. Kannan K, Inagaki A, Silber J, Gorovets D, Zhang J, Kastenhuber ER, Heguy A, Petrini JH, Chan TA, Huse JT. Whole-exome sequencing identifies ATRX mutation as a key molecular determinant in lower-grade glioma. Oncotarget. 2012; 3:1194-1203.

2. Wen PY, Kesari S. Malignant gliomas in adults. The New England journal of medicine. 2008; 359:492-507.

3. Yan H, Parsons DW, Jin G, McLendon R, Rasheed BA, Yuan W, Kos I, Batinic-Haberle I, Jones S, Riggins GJ, Friedman H, Friedman A, Reardon D, Herndon J, Kinzler KW, Velculescu VE, et al. IDH1 and IDH2 mutations in gliomas. The New England journal of medicine. $2009 ; 360: 765-773$.

4. Olar A, Wani KM, Alfaro-Munoz KD, Heathcock LE, van Thuijl HF, Gilbert MR, Armstrong TS, Sulman EP, Cahill DP, Vera-Bolanos E, Yuan Y, Reijneveld JC, Ylstra B, Wesseling P, Aldape KD. IDH mutation status and role of WHO grade and mitotic index in overall survival in grade II-III diffuse gliomas. Acta Neuropathol. 2015; 129:585-596.

5. Louis DN, Ohgaki H, Wiestler OD, Cavenee WK, Burger PC, Jouvet A, Scheithauer BW, Kleihues P. The 2007 WHO classification of tumours of the central nervous system. Acta neuropathologica. 2007; 114:97-109.

6. Gorovets D, Kannan K, Shen R, Kastenhuber ER, Islamdoust $\mathrm{N}$, Campos $\mathrm{C}$, Pentsova $\mathrm{E}$, Heguy A, Jhanwar SC, Mellinghoff IK, Chan TA, Huse JT. IDH mutation and neuroglial developmental features define clinically distinct subclasses of lower grade diffuse astrocytic glioma. Clinical cancer research : an official journal of the American Association for Cancer Research. 2012; 18:2490-2501.

7. Cai J, Yang P, Zhang C, Zhang W, Liu Y, Bao Z, Liu X, Du W, Wang H, Jiang T, Jiang C. ATRX mRNA expression combined with IDH1/2 mutational status and Ki-67 expression refines the molecular classification of astrocytic tumors: evidence from the whole transcriptome sequencing of 169 samples samples. Oncotarget. 2014; 5:2551-2561.

8. Liu Y, Tang K, Yan W, Wang Y, You G, Kang C, Jiang T, Zhang W. Identifying Ki-67 specific miRNA-mRNA interactions in malignant astrocytomas. Neuroscience letters. 2013; 546:36-41.

9. Jin Q, Zhang W, Qiu XG, Yan W, You G, Liu YW, Jiang $\mathrm{T}$, Wang L. Gene expression profiling reveals Ki-67 associated proliferation signature in human glioblastoma. Chinese medical journal. 2011; 124:2584-2588.

10. Colman H, Giannini C, Huang L, Gonzalez J, Hess K, Bruner J, Fuller G, Langford L, Pelloski C, Aaron J, Burger P, Aldape K. Assessment and prognostic 
significance of mitotic index using the mitosis marker phospho-histone $\mathrm{H} 3$ in low and intermediate-grade infiltrating astrocytomas. The American journal of surgical pathology. 2006; 30:657-664.

11. Hartmann C, Hentschel B, Wick W, Capper D, Felsberg J, Simon M, Westphal M, Schackert G, Meyermann R, Pietsch T, Reifenberger G, Weller M, Loeffler M, von Deimling A. Patients with IDH1 wild type anaplastic astrocytomas exhibit worse prognosis than IDH1-mutated glioblastomas, and IDH1 mutation status accounts for the unfavorable prognostic effect of higher age: implications for classification of gliomas. Acta neuropathologica. 2010; 120:707-718.

12. Thon N, Eigenbrod S, Kreth S, Lutz J, Tonn JC, Kretzschmar H, Peraud A, Kreth FW. IDH1 mutations in grade II astrocytomas are associated with unfavorable progression-free survival and prolonged postrecurrence survival. Cancer. 2012; 118:452-460.

13. Cohen AL, Holmen SL, Colman H. IDH1 and IDH2 mutations in gliomas. Current neurology and neuroscience reports. 2013; 13:345.

14. Weller M, Stupp R, Hegi ME, van den Bent M, Tonn JC, Sanson M, Wick W, Reifenberger G. Personalized care in neuro-oncology coming of age: why we need MGMT and $1 \mathrm{p} / 19 \mathrm{q}$ testing for malignant glioma patients in clinical practice. Neuro-oncology. 2012; 14:iv100-108.

15. Turcan S, Rohle D, Goenka A, Walsh LA, Fang F, Yilmaz E, Campos C, Fabius AW, Lu C, Ward PS, Thompson CB, Kaufman A, Guryanova O, Levine R, Heguy A, Viale A, et al. IDH1 mutation is sufficient to establish the glioma hypermethylator phenotype. Nature. 2012; 483:479-483.

16. Dang L, White DW, Gross S, Bennett BD, Bittinger MA, Driggers EM, Fantin VR, Jang HG, Jin S, Keenan MC,
Marks KM, Prins RM, Ward PS, Yen KE, Liau LM, Rabinowitz JD, et al. Cancer-associated IDH1 mutations produce 2-hydroxyglutarate. Nature. 2009; 462:739-744.

17. Zhang C, Moore LM, Li X, Yung WK, Zhang W. IDH1/2 mutations target a key hallmark of cancer by deregulating cellular metabolism in glioma. Neuro-oncology. 2013; 15:1114-1126.

18. Horbinski C. What do we know about IDH1/2 mutations so far, and how do we use it? Acta neuropathologica. 2013; 125:621-636.

19. Lass U, Numann A, von Eckardstein K, Kiwit J, Stockhammer F, Horaczek JA, Veelken J, Herold-Mende C, Jeuken J, von Deimling A, Mueller W. Clonal analysis in recurrent astrocytic, oligoastrocytic and oligodendroglial tumors implicates IDH1- mutation as common tumor initiating event. PloS one. 2012; 7:e41298.

20. Yan W, Zhang W, You G, Bao Z, Wang Y, Liu Y, Kang C, You Y, Wang L, Jiang T. Correlation of IDH1 mutation with clinicopathologic factors and prognosis in primary glioblastoma: a report of 118 patients from China. PloS one. 2012; 7:e30339.

21. Zhang CB, Bao ZS, Wang HJ, Yan W, Liu YW, Li MY, Zhang W, Chen L, Jiang T. Correlation of IDH1/2 mutation with clinicopathologic factors and prognosis in anaplastic gliomas: a report of 203 patients from China. Journal of cancer research and clinical oncology. 2014; 140:45-51.

22. Niikura N, Sakatani T, Arima N, Ohi Y, Honma N, Kanomata N, Yoshida K, Kadoya T, Tamaki K, Kumaki N, Iwamoto T, Sugie T, Moriya T. Assessment of the Ki67 labeling index: a Japanese validation ring study. Breast cancer. 2014 May 3. PMID: 24794952. 